\section{Direct investigation of the Aspergillus GDP-mannose transporter by STD NMR spectroscopy}

\section{Andrea Maggioni ${ }^{\ddagger}$, Julian Meier ${ }^{\ddagger}$, Françoise Routier, Thomas Haselhorst, Joe Tiralongo*[a]}

Aspergillus species, predominantly A. fumigatus and A. flavus, represent some of the most important air-borne fungal pathogens world-wide. Found ubiquitously in nature, Aspergillus spp. cause a variety of diseases depending on the immune status of the host. ${ }^{[1]}$ Most cases of invasive aspergillosis (IA) are associated with HIV infections, haematological malignancies, leukaemia or lymphoma. ${ }^{[2]}$ The harmless status of Aspergillus has changed over the past two decades, with the frequency of IA increasing 14-fold in the past 10 years. ${ }^{[3]}$ IA has a mortality of over $95 \%$, and the fungus is able to cause several forms of disease in humans of which IA is the most severe. The high mortality rate of this disease warrants increased efforts towards understanding the basic principles of Aspergillus pathogenicity. ${ }^{[1,4]}$ New antifungal agents have aided in both the treatment of fungal infections and in the prevention of disease in susceptible individuals, but resistance is an increasing concern. ${ }^{[5]}$ Only a few potential antifungal targets have been exploited to date and there is a critical need for the discovery and development of novel antifungal agents that will result in improved therapy in this ever-expanding patient population.

A novel class of antifungal agents called the echinocandins target the synthesis of $\beta$-(1,3)-glucan, the a major cell wall component (galactomannan, Figure 1a). ${ }^{[6]}$ Galactomannan is composed of a linear mannan core branched with short $\square 1,5$-linked galactofuranose chains, bound covalently to the cell wall $\beta$-(1,3)-glucan, anchored to the lipid membrane by a glycosylphosphatidylinositol (GPI), or released in the environment during tissue invasion or growth in culture. $\left.^{[7,}{ }^{8}\right]$ Besides being an abundant component of the extracellular matrix, secreted galactomannans are a serological diagnostic of IA. ${ }^{[9,10]}$ The efficient biosynthesis of the fungus galactomannan cell wall requires the transport of nucleotide sugars, the donor substrates for glycosyltransferases, from the cytosol into the Golgi lumen.

[a] Andrea Maggioni, Julian Meier, Dr Thomas Haselhorst and Dr Joe Tiralongo ( ${ }^{*}$ Corresponding Authors)

Institute for Glycomics, Gold Coast Campus, Griffith University, Queensland, 4222, Australia

Fax: (+) 61(0)7555 28098

E-mail:j.tiralongo@griffith.edu.au

[b] Prof Francoise Routier, Institut für Zelluläre Chemie, Zentrum Biochemie, Medizinische Hochschule Hannover, Carl-Neuberg Strasse 1, 30625 Hannover, Germany

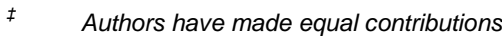

Supporting information for this article is available on the WWW under http://www.chembiochem.org or from the author
This critical task is carried out by a group of proteins termed nucleotide-sugar transporters (NSTs). ${ }^{[1]}$ Some NST activities are found in all organisms, however some are restricted to certain lineages. ${ }^{[12,13]}$ For example, a Golgi resident GDP-mannose (GDPMan) transporter (Figure 1b) is not found in mammalian cells but in yeast, Leishmania, and Aspergillus. ${ }^{[12,13]}$ Since NST activity is required prior to glycosylation, there has been great interest in better understanding NST specificity, regulation, and cellular targeting. At present, there is relatively little information on the structure of the NST protein complex, the basis for NST specificity, and the targeting and organization of NSTs within the cell. Loss of expression of all Man-containing molecules in A. fumigatus by a targeted deletion of the genes involved in GDP-Man biosynthesis resulted in complete loss of virulence in macrophages and susceptible mice. ${ }^{[14,15]}$ In addition a more recent study has shown that the GDP-Man transporter (encoded by the gmtA gene) is essential in Aspergillus niger. ${ }^{[16]}$ Therefore, the Aspergillus GDPMan transporter (AspGMT), which is not found in mammalian cells, $[12,13]$ represents a promising drug target for the development of novel anti-fungal drugs that inhibit GDP-Man translocation into the Golgi lumen required for the biosynthesis of the fungal virulence factor galactomannan.

NST's are Golgi- or Endoplasmic Reticulum (ER)-resident type III transmembrane proteins with 8-10 predicted membrane-spanning domains. There is currently no 3D- or 2D-crystal structure available for any known NST. Therefore, a conventional structure-based drug design approach is currently not feasible. Work previously performed in our laboratory showed that it is possible to detect the binding of nucleotide sugars to endogenous NSTs present in isolated Golgi-enriched fractions $(\mathrm{GeFs})$ isolated by $P$. pastoris. ${ }^{[17]}$ Here we report the first direct investigation of AspGMT substrate binding by STD NMR spectroscopy and the identification of important moieties critical for AspGMT interaction with GDP-Man. This information will significantly assist the design of novel inhibitors of the AspGMT, and potentially galactomannan synthesis.

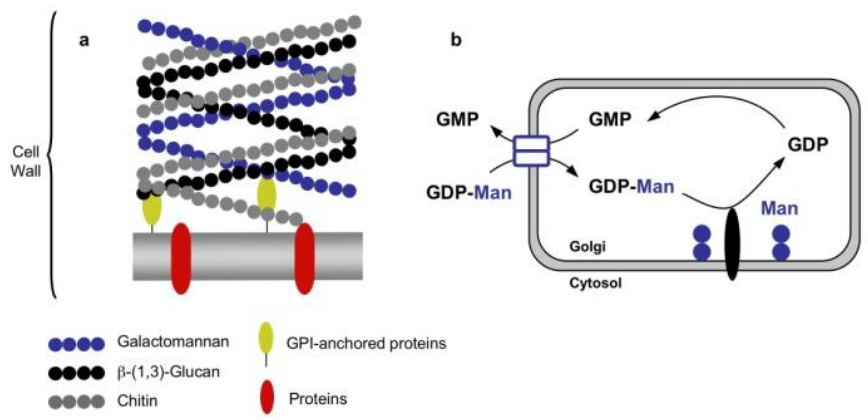

Figure 1. (a) The cell wall of Aspergillus sp. is composed of $\beta-(1,3)$-glucan, chitin and galactomannan. Galactomannan is covalently attached to $\beta-(1,3)$ glucan or anchored to the lipid membrane through a GPI anchor. (b) The biosynthesis of the fungus galactomannan cell wall requires the transport of GDP-Man, the donor substrates for mannosyltransferases, from the cytosol into the Golgi lumen. GDP-Man transported into the Golgi is coupled with the export of GMP in an antiporter mechanism. 


\section{GDP-Mannose interacts with the Aspergillus GDP. Mannose Transporter}

Based on our initial observation that binding of nucleotide sugars to endogenous NSTs present in isolated GeFs can be monitored by STD NMR spectroscopy, ${ }^{[17]}$ we focused on investigating the binding of GDP-Man to GeFs isolated from Aspergillus (Asp-GeF). Our aim was firstly to determine if it is possible to detect ligand binding to NSTs that are present in $A s p-G e F s$ at endogenous levels, and secondly to establish a preliminary binding/affinity model for GDPMan interaction with the AspGMT.
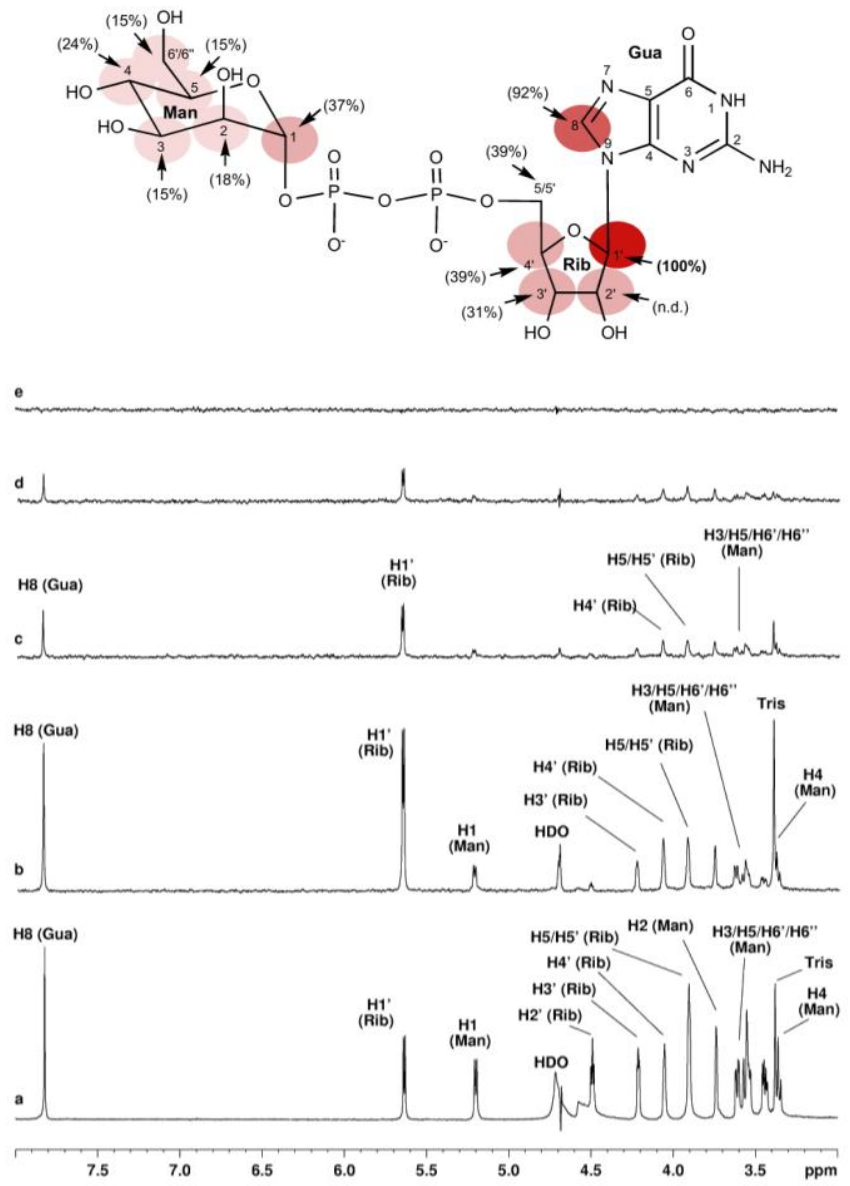

Figure 2. ${ }^{1} \mathrm{H}$ (a) and STD NMR spectra (b-e) of GDP-Man in complex with AspGeF (b), Asp-GeF-DN (c), human-GeF (d) and no protein (e). The onresonance frequency was set to $-1.00 \mathrm{ppm}$ and the off-resonance to $300 \mathrm{ppm}$. The total saturation was $3 \mathrm{sec}$. The residual water signal was supressed using a gradient selective WATERGATE sequence. All NMR experiments were performed at $600 \mathrm{MHz}$ and $283 \mathrm{~K}$ in $10 \mathrm{mM}$ deuterated TRIS containing $2 \mathrm{mM}$ $\mathrm{MgCl}_{2}, \mathrm{pH}$ 7.5. The uncorrected STD spectrum (b) was used to determine the epitope map and relative STD NMR effects were calculated according to the equation $A_{S T D}=\left(\begin{array}{lll}I_{0} & \times & I_{\text {sat }}\end{array}\right) / I_{0}=I_{S T D} / I_{0}$.

Figure 2 shows the ${ }^{1} \mathrm{H}$ (Figure 2a) and STD NMR spectra of GDP-Man in the presence of Golgi preparation from Aspergillus (Asp-GeF) (Figure 2b). It is immediately apparent, due to strong STD NMR signals that GDP-Man binds to proteins present in the Golgi preparation. Most striking is the observation that the $\mathrm{H} 1$ proton of the ribose moiety received the most saturation and hence was set at $100 \%$ to calculate relative STD NMR intensities. The importance of the $\mathrm{H} 1$ proton of the ribose moiety for the interaction of nucleotide sugars with their corresponding binding protein has been previously reported and our finding is in good agreement with the literature. ${ }^{[17-20]}$

To address the question of whether the observed interaction of GDP-Man with $A s p-G e F$ is due to binding of the endogenous GMT, a number of control NMR experiments were performed. Figure $2 \mathrm{c}$ shows a clear decrease in STD signals upon denaturing of protein associated with $A s p-\mathrm{GeF}$ (Asp-GeF-DN) by heating the Golgi preparation to $70{ }^{\circ} \mathrm{C}$ for 15 min prior to the addition of GDP-Man. However, residual STD signals remained suggesting either incomplete denaturation or non-specific binding to the Golgi membrane. To investigate this further, the binding of GDP-Man to GeFs prepared from the human cell line HT-29 (human-GeF; mammalian cells are know not to express a $\mathrm{GMT}^{[12]}$ ) was studied. Figure $2 \mathrm{~d}$ shows that GDP-Man complexed with human-GeF gave similar STD signal intensities to that observed for Asp-GeF-DN. This suggests that the STD signals observed using Asp-GeF represented the interaction of GDP-Man to the endogenous Aspergillus GMT (AspGMT). However, the procedure for preparation GeFs can occasionally lead to the production of insideout vesicles where proteins that normally face the Golgi lumen (inside) now face the cytosol (outside). Therefore, mannosyltransferases that catalyses the transfer of Man from GDPMan to glycoconjugates (Figure 1b), which would normally face the lumen, could become exposed in inside-out GeFs. To rule out the possibility that the observed STD effect was due to the interaction of GDP-Man with mannosyltransferases, a GDP-Man consumption assay was performed. In this experiment if GDP-Man was able to interact with an exposed mannosyltransferase, GDP-Man consumption over time should be observable Asp-GeFs were incubated with GDP-Man for $2 \mathrm{~h}$ at $37^{\circ} \mathrm{C}$, and the consumption of GDP-Man monitored in real time by ${ }^{1} \mathrm{H}$ NMR spectroscopy. The ${ }^{1} \mathrm{H}$ NMR spectrum of GDP-Man in the presence and absence of Asp$\mathrm{GeF}$ at $37{ }^{\circ} \mathrm{C}$ clearly shows that GDP-Man was not consumed, and therefore it is unlikely that GDP-Man was interacting with exposed mannosyltransferases (see supporting information). Our data strongly suggest that GDP-Man binds specifically to the endogenous AspGMT. The low STD NMR signal intensities that were observed for GDP-Man in complex with denatured Golgi fractions (Asp-GeFDN) are likely a direct result of an incomplete denaturation of Golgi proteins. On the contrary, the residual STD NMR signals of the GDP-Man:human-GeF complex (Figure 2d) are probably the result GDP-Man binding to other non-GMT proteins. Therefore, both STD NMR control experiments cannot be used to generate double difference STD NMR spectra (STDD) to eliminate background binding. A more suitable control experiment would be the complexing of GDP-Man with GeF's derived from an Aspergillus GMT deficient mutant. However, Carvalho and co-workers have shown recently ${ }^{[16]}$ that a knock-out deletion of the GMT gene in Aspergillus niger is lethal. Therefore we determined the binding epitope for the interaction of GDP-Man with the Asp GMT (Figure 2 upper panel) using the uncorrected STD NMR spectrum as shown in Figure $2 \mathrm{~b}$. In addition we also generated two STDD NMR spectra. The first was obtained by subtracting the GDP-Man:Asp-GeF-DN complex (Figure 2c) from the spectrum obtained for the GDPMan:Asp-GeF complex (Figure 2b), and the second by subtracting the GDP-Man:Asp-GeF-DN complex (Figure 2c) from the spectrum obtained for the GDP-Man:human-GeF complex (Figure 2d) (see supporting information). A comparison of relative STD NMR effects reveals that the epitope map obtained from the uncorrected STD NMR spectrum differs only marginal from an epitope map based on both STDD NMR spectra (see Table S2, supporting information).

The nucleotide sugar interactome reveals that the nucleotide portion of GDP-Man receives the greatest amount of saturation 
relative to the sugar moiety, suggesting that the GDP moiety is strongly interacting with $A s p$ GMT. However, some saturation is also being received by the mannose (Man) component, indicating that the sugar moiety is likewise important for binding. A similar observation has also been reported for the interaction of CMP-sialic acid with CMP-sialic acid transporter, ${ }^{[17]}$ and points to a key role for the sugar moiety in mediating NST specificity.

GDP, GMP and Mannose compete with GDP-Mannose for the Aspergillus GDP-Mannose Transporter binding site.

To further probe the interaction of nucleotide sugars and nucleotides with the $A s p \mathrm{GMT}$ competition STD NMR experiments using GMP-Man, GMP, GDP and Man were acquired. First, a STD NMR spectrum of GDP-Man in the presence of $A s p-\mathrm{GeF}$ was acquired followed by the consecutive addition of GMP and GDP. The extent of competition was determined by measuring the reduction in signal intensity of the $\mathrm{H} 8$ proton of the guanidine residue of GDP-Man. For example, this specific STD NMR signal (H8 Gua, GDP-Man) is reduced by $46 \%$ following the addition of an equimolar concentration of GMP (Figure 3a). A further addition of GDP to the Asp-GeF:GDP-Man:GMP mixture revealed that the STD NMR signal intensity of the $\mathrm{H} 8$ proton of the guanidine residue of GDP-Man was reduced by an additional $29 \%$, leading to a total decrease of $75 \%$ in comparison to the STD spectrum acquired with only GDP-Man. Interestingly, an identical decrease of all GDP-Man STD NMR specific signals was also detected (Gua H8, 7.85 ppm; Man H1, 5.25 ppm; Man H2 3.75 ppm; and Man H3-H6, 3.7-3.4 ppm, see supporting information). This observation clearly suggests that GMP and GDP are able to displace GDP-Man, and thus GDPMan, GMP and GDP are binding to the same site of the AspGMT in a competitive manner. This is supported by the fact that the first addition of an equimolar amount of GMP lead to a mean reduction in GDP-Man specific signals by $47 \pm 1.4 \%$ (see supporting information). This compares favourably with the expected $50 \%$ reduction if GDP-Man and GMP competed for the same binding site with equal affinity. The subsequent addition of GDP to GDP-Man and GMP lead to a total reduction in GDP-Man specific signals by $77 \pm 2.1 \%$ (see supporting information). However, in this instance the mean reduction in GDP-Man specific signals is greater than the $66 \%$ expected if all three ligands interacted with equal affinity. Our data suggests that GDP may bind to the AspGMT with higher affinity because its addition resulted in a greater displacement of GDP-Man than would be expected.

The effect of GMP and GDP on GDP-Man interaction with Asp$\mathrm{GeF}$, lead us to explore the effect of D-mannose (D-Man) on GDPMan binding in a subsequent STD NMR competition experiment. Figure $3 b$ shows the competitive effects of D-Man on the binding of GDP-Man. A small reduction in signal intensity of the H8 proton of the guanidine residue of GDP-Man (about $\sim 15 \%$ ) indicates that DMan does not readily displace the tightly bound GDP-Man sugar nucleotide, suggesting only a weak interaction of Man with the AspGMT. Interestingly, STD signals of D-Man (H1 Man) are detectable, although with very low intensity (see supporting information). It is important to note that D-Man in solution exists as a mixture of $67 \% \alpha$-D-Man and $33 \% \beta$-D-Man resulting in a nonequimolar mix. Therefore, it is likely that the actual displacement potency of D-Man is higher than the measured $15 \%$.
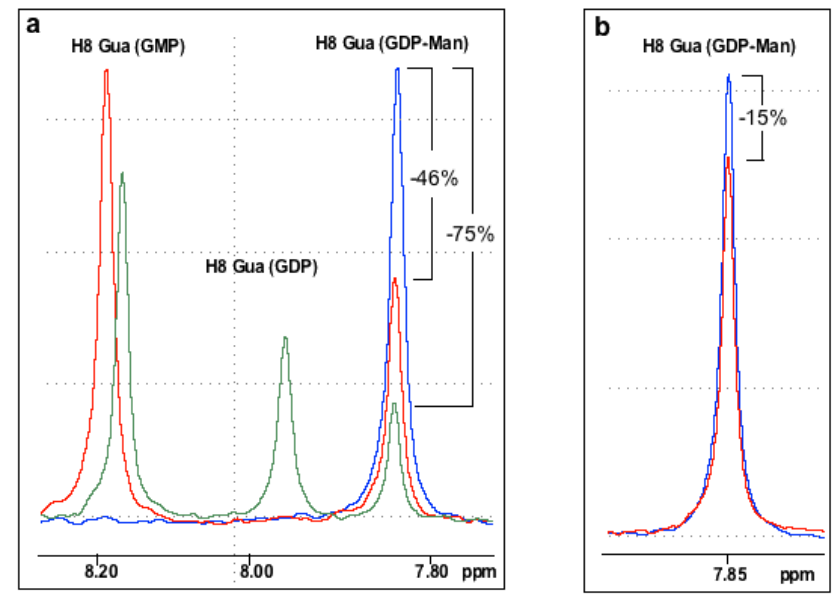

Figure 3. Absolute STD NMR signal intensities of the $\mathrm{H} 8$ proton of the guanidine residue of GDP-Man in complex with Asp-GeF (a and b, blue spectrum), after addition of an equimolar concentration of GMP (a, red spectrum), GDP (a, green spectrum) and mannose ( $b$, red spectrum)

The data present herein shows for the first time the direct analysis of nucleotide sugar, nucleotide and free sugar interactions with the Aspergillus GDP-Man transporter (AspGMT), a protein that represents a potential novel target for drug discovery. We revealed through competition STD NMR experiments that GDP appears to bind tighter to the AspGMT than GMP and GDP-Man, with Man found to be the weakest binding ligand. Taken together our data permits the relative importance/affinity of individual ligand moieties that bind AspGMT to be summarized as follows:

$$
\mathrm{GDP} \geq \mathrm{GMP} \simeq \mathrm{GDP}-\mathrm{Man}>>\mathrm{Man}
$$

As shown in Figure $1 \mathrm{~b}$ the natural antiporter substrates for the GMT are GDP-Man and GMP, and our data showed that both make intimate contacts with the AspGMT binding site. However, the observation that in fact GDP may bind the GMT with higher affinity than the natural substrates can now be exploited in the design of novel GMT inhibitors.

\section{Experimental Section}

General - GDP-Man, GMP, GDP and $\mathrm{D}_{2} \mathrm{O}$ (99.9\%) were purchased from Sigma Aldrich (Australia).

Aspergillus culture and subcellular fractionation - Aspergillus niger ATCC 1004 were cultured on complete-agar YMPG media $(0.3 \% \mathrm{w} / \mathrm{v}$ yeast extract, $0.3 \% \mathrm{w} / \mathrm{v}$ malt extract, $0.5 \% \mathrm{w} / \mathrm{v}$ peptone, $1 \% \mathrm{w} / \mathrm{v}$ glucose, and $1.5 \% \mathrm{w} / \mathrm{v}$ agar) for 10 days at $25{ }^{\circ} \mathrm{C}$ in $75 \mathrm{~cm}^{2}$ tissue culture flasks fitted with filtered lids. Spores were harvested as described by Tiralongo et al. $2009^{[21]}$ with glass beads in the presence of $20 \mathrm{mM}$ PBS, pH 7.4 containing $0.05 \%$ Tween-20 (PBST) by vigorous shaking. Subsequently, the dislodged spores were filtered through sterile glass wool, collected by centrifugation (1000 g, $5 \mathrm{~min}$ ), resuspended in $20 \mathrm{mM}$ PBS and counted. Liquid YMPG cultures ( $1 \mathrm{~L}$ ) were inoculated with $0.5-2 \times 10^{6}$ spores $/ \mathrm{mL}$ and incubated for $19 \mathrm{~h}$ at $37^{\circ} \mathrm{C}$ with shaking at $150 \mathrm{rpm}$. The mycellium was harvested and Golgi-enriched fractions isolated according to Record et $a l^{[22]}$ and Engel et al. ${ }^{[23]}$ Briefly, the mycellium was collected on Whatman no. 4 filter paper washed with milliQ water, removed from the filter paper and weighed. The mycellium was ground in a mortar with $1-1.5$ volumes of sterile sand and $30 \mathrm{~mL}$ of 
lysis buffer (10 mM HEPES/Tris, pH 7.4, 0.8 M sorbitol, $1 \mathrm{mM}$ EDTA, supplemented with EDTA-free Protease Inhibitor (Roche). Large particles were removed by filtration through plain gauze that was rinsed with $20-50 \mathrm{~mL}$ lysis buffer and subsequently squeezed to recover all lysed material. The filtrate was centrifuged at $10,000 \mathrm{~g}$ for $30 \mathrm{~min}$, with the resulting supernatant further centrifuged at $27,000 \mathrm{~g}$ for $20 \mathrm{~min}$. A Golgi-enriched fraction $(\mathrm{GeF})$ was isolated from supernatant by ultracentrifugation at $100,000 \mathrm{~g}$ for $45 \mathrm{~min}$. The resulting pellet was resupended in $800 \mu \mathrm{L}$ of lysis buffer, frozen using liquid nitrogen in $100 \mu \mathrm{L}$ aliquots and stored at $-80{ }^{\circ} \mathrm{C}$ until required.

NMR experiments - All NMR experiments were performed on a Bruker Avance $600 \mathrm{MHz}$ NMR spectrometer, equipped with a $5 \mathrm{~mm}$ cryoprobe using Shigemi tubes. Chemical shifts of GDP-Man, GDP and GMP were assigned using COSY, TOCSY and HSQC experiments.

STD NMR experiments - STD NMR experiments were performed essential as described by Maggioni et al. 2008. ${ }^{[17]}$ The frozen GeFs were thawed slowly on ice and buffer exchanged. Briefly, $100-200 \mu \mathrm{L}$ of NMR buffer $(10 \mathrm{mM}$ deuturated Tris/DCl $\mathrm{pH} 7.5$, supplemented with $2 \mathrm{mM} \mathrm{MgCl}_{2}$ in $\mathrm{D}_{2} \mathrm{O}$ ) was used to suspend the GeFs. Following centrifugation at $18,000 \mathrm{x} g$ for $10 \mathrm{~min}$ the supernatant was removed, the pellet resuspended in NMR buffer, and centrifugation repeated. This procedure was performed twice more to assure that the exchange from lysis-buffer to NMR-buffer was complete. All NMR spectra were acquired using a Bruker 600 $\mathrm{MHz}$ ultrashield NMR apparatus equipped with a ${ }^{1} \mathrm{H} /{ }^{15} \mathrm{~N} /{ }^{13} \mathrm{C}$ cryoprobe. NMR signal assignment of GDP-Man, GDP, GMP was achieved by

Each STD NMR experiment was performed in Shigemi NMR tubes containing $200 \mu \mathrm{g}$ equivalent of protein as GeF's in NMR buffer and 100-fold molar excess of ligand ( $200 \mu \mathrm{L}$ final volume). To monitor any changes in the ligand during an STD NMR acquisition, proton spectra before and after the STD NMR acquisition was made using 8 scans each. Protein saturation was achieved using a Gaussian pulse train cascade for $3 \mathrm{sec}$ at the onresonance frequency of $-1 \mathrm{ppm}$ and at the off -resonance frequency of $300 \mathrm{ppm}$. To suppress residual HDO signals water suppression by gradient tailored excitation (WATERGATE) sequence was used. A total of 1024 scans $(1 \mathrm{~K})$ were acquired. The on- and off- resonance spectra FIDs were stored and processed separately and the subtraction of the on-resonance and the off-resonance spectrum resulted in the STD NMR spectrum. To determine specific binding of ligands to protein saturation transfer double difference (STDD) spectra were generated by superimposing two individual STD NMR spectra followed by a subtraction. STDD NMR spectra were used to calculate the relative STD NMR effects according to the formula
$A_{S T D}=\left(I_{0} \times I_{\text {sat }}\right) / I_{0}=I_{S T D} / I_{0}$. using the $\mathrm{H} 1$ proton of the Ribose (H1 Rib) as $100 \%$.

Monitoring GDP-Man consumption by NMR spectroscopy The consumption of GDP-Man was determined using a ${ }^{1} \mathrm{H}-$ acquisition with 16 scans following incubation for $2 \mathrm{~h}$ at $37^{\circ} \mathrm{C}$ in the presence and absence of Asp-GeF.

Keywords: GDP-Mannose transporter, Aspergillus, Fungi, Saturation Transfer Difference (STD) NMR, Nucleotide sugar transporters

Received: ((will be filled in by the editorial staff))

Published online: ((will be filled in by the editorial staff)

[1] T. R. Dagenais, N. P. Keller, Clin Microbiol Rev 2009, 22, 447

[2] R. Ben-Ami, R. E. Lewis, D. P. Kontoyiannis, Br J Haematol 2010, 150, 406 .

[3] A. H. Groll, P. M. Shah, C. Mentzel, M. Schneider, G. Just-Nuebling, K. Huebner, J Infect 1996, 33, 23.

[4] A. Abad, J. V. Fernandez-Molina, J. Bikandi, A. Ramirez, J. Margareto, J. Sendino, F. L. Hernando, J. Ponton, J. Garaizar, A. Rementeria, Rev Iberoam Micol 2010, 27, 155.

[5] S. J. Howard, M. C. Arendrup, Med Mycol 2011, 49 Suppl 1, S90.

[6] N. D. Grover, Indian J Pharmacol 2010, 42, 9.

[7] A. Gastebois, C. Clavaud, V. Aimanianda, J. P. Latge, Future Microbiol 2009, 4, 583

[8] J. P. Latge, Mol Microbiol 2007, 66, 279

[9] F. M. Marty, S. Koo, Med Mycol 2009, 47 Suppl 1, S233.

[10] A. Kedzierska, Pol J Microbiol 2007, 56, 3.

[11] C. E. Caffaro, C. B. Hirschberg, Acc Chem Res 2006, 39, 805

[12] I. Martinez-Duncker, R. Mollicone, P. Codogno, R. Oriol, Biochimie 2003, 85, 245.

[13] N. Ishida, M. Kawakita, Pflugers Arch 2004, 447, 768

[14] A. Kotz, J. Wagener, J. Engel, F. Routier, B. Echtenacher, A. Pich, M. Rohde, P. Hoffmann, J. Heesemann, F. Ebel, Fungal Genet Biol 2010, 47, 169.

[15] J. Wagener, B. Echtenacher, M. Rohde, A. Kotz, S. Krappmann, J. Heesemann, F. Ebel, Eukaryot Cell 2008, 7, 1661

[16] N. D. Carvalho, M. Arentshorst, X. O. Weenink, P. J. Punt, C. A. van den Hondel, A. F. Ram, Fungal Biol. 2011, 115, 253

[17] A. Maggioni, M. von Itzstein, J. Tiralongo, T. Haselhorst, Chembiochem 2008, 9, 2784 .

[18] J. Angulo, C. Rademacher, T. Biet, A. J. Benie, A. Blume, H. Peters, M. Palcic, F. Parra, T. Peters, Methods Enzymol 2006, 416, 12.

[19] A. C. Lamerz, T. Haselhorst, A. K. Bergfeld, M. von Itzstein, R. Gerardy-Schahn, J Biol Chem 2006, 281, 16314.

[20] R. Bohm, F. Freiberger, K. Stummeyer, R. Gerardy-Schahn, M. von Itzstein, T. Haselhorst, Chembiochem 2009, 11, 170.

[21] J. Tiralongo, T. Wohlschlager, E. Tiralongo, M. J. Kiefel, Microbiology 2009, 155, 3100 .

[22] E. Record, M. Asther, S. Moukha, D. Marion, V. Burlat, K. Ruel, M. Asther, Can J Microbiol 1998, 44, 945.

[23] J. Engel, P. S. Schmalhorst, T. Dork-Bousset, V. Ferrieres, F. H. Routier, J Biol Chem 2009. 


\section{Entry for the Table of Contents}

Layout 1:

COMMUNICATIONS

Here we report on the direct analysis of the GDP-Man transporter in isolated Aspergillus Golgi-enriched fractions by STD NMR spectroscopy. STD NMR spectra reveal that the guanidine and ribose moiety of GDP-Man receives more saturation than the mannose sugar residue. Competition STD NMR experiments revealed that GDP appears to bind tighter to the GDPMan transporter than GMP and GDP-Man, with Man found to be the weakest binding ligand. Taken together our data permits the relative importance/affinity of individual ligand moieties that bind the Aspergillus GDP-Man transporter to be summarized as follows:

$\mathrm{GDP} \geq \mathrm{GMP} \simeq$ GDP-Man $>>$ Man .

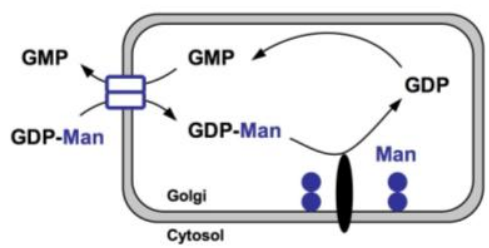

Andrea Maggioni, Julian Meier, Francoise Routier, Thomas Haselhorst, Joe Tiralongo*

Page No. - Page No.

Direct investigation of Aspergillus GDP-mannose transporter by STD NMR spectroscopy.

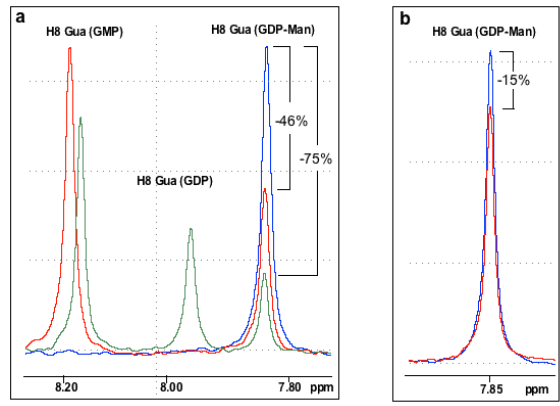




\section{Supporting Information}

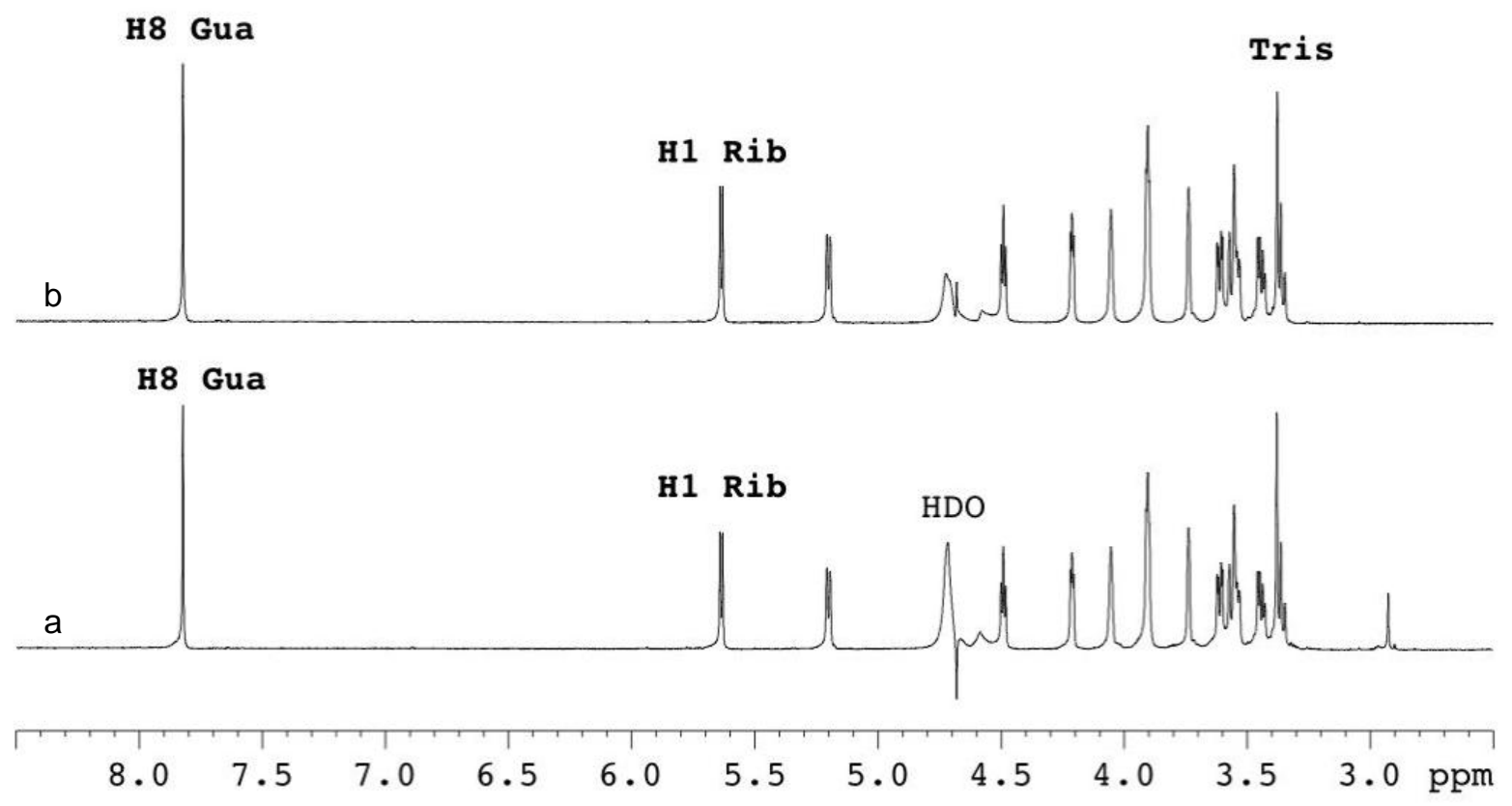

Figure S1. ${ }^{1} \mathrm{H}$ NMR spectra of GDP-Man (a) and GDP-Man incubated for $2 \mathrm{~h}$ at $37^{\circ} \mathrm{C}$ in the presence of Asp-GeF (b). The spectra clearly indicate that GDP-Man was not being consumed.

Table S1. Apart from H8 Gua signal of GDP-Man, the extent of competition was also determined by measuring the reduction of STD NMR signal intensity of the $\mathrm{H} 1, \mathrm{H} 2$ and $\mathrm{H} 3-$ $\mathrm{H} 6$ protons of the mannose moiety. The mean reduction and standard deviation (SD) across all these signals are shown. The addition of equimolar GMP led to a mean reduction in GDPMan signals by $47 \pm 1.4 \%$. The subsequent addition of GDP to GDP-Man and GMP led to a total reduction in GDP-Man specific signals by $77 \pm 2.1 \%$

\% Reduction in GDP-Man specific STD signals

\begin{tabular}{lcccccc}
\hline $\begin{array}{l}\text { Competing } \\
\text { nucleotide }\end{array}$ & $\begin{array}{c}\text { H8 Gua } \\
(7.85 \mathrm{ppm})\end{array}$ & $\begin{array}{c}\text { H1 Man } \\
(5.25 \mathrm{ppm})\end{array}$ & $\begin{array}{c}\text { H2 Man } \\
(3.75 \mathrm{ppm})\end{array}$ & $\begin{array}{c}\text { H3-H6 Man } \\
(3.7-3.4 \mathrm{ppm})\end{array}$ & Mean & SD \\
\hline \hline GMP & 46 & 49 & 46 & 46 & 47 & 1.4 \\
GMP + GDP & 75 & 79 & 75 & 78 & 77 & 2.1 \\
\hline
\end{tabular}




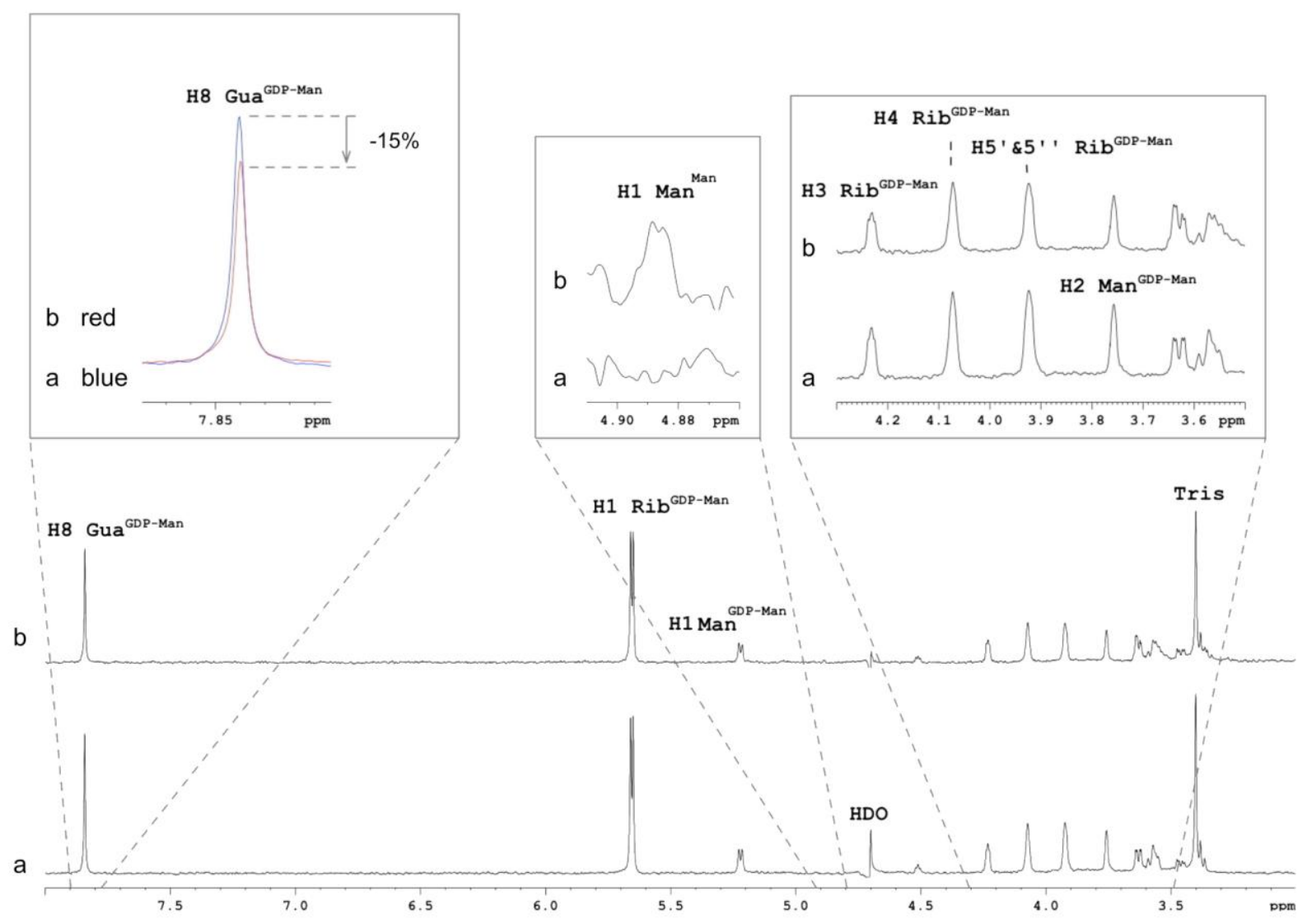

Figure S2. a) STD NMR spectra acquired of GDP-Man complexed with Asp-GeF. b) STD NMR spectra acquired of GDP-Man complexed with Asp-GeF following the addition of equimolar Man. For the $\mathrm{H} 8$ Gua signals and for the $\mathrm{H} 3, \mathrm{H} 4$, and $\mathrm{H} 5$ Rib signals a reduction in STD signal intensity is visible. The only STD NMR signal of the free mannose that could be detected is the $\mathrm{H} 1$ (a-anomer) signal (4.89 ppm), although with very low intensity.

Table S2. Relative STD NMR values of GDP-Man. The STD NMR spectrum of the AspGEF:GDP-Man complex was used to calculate the relative STD NMR effects for GDPMan. STDD spectra (Asp-GeF:GDP-Man - Asp-GeF-DN:GDP-Man) and (Asp-GeF:GDPMan - human-GeF:GDP-Man) were also used to calculate relative STD NMR effects for GDP-Man. All STD NMR effects were calculated according to the formula $A$ STD $=(10 x$ $\left.I_{\text {sat }}\right) / I_{0}=I_{\text {sTD }} / I_{0}$ using the $\mathrm{H} 1$ ' (Rib) proton to $100 \%$.

\begin{tabular}{|c|c|c|c|c|c|c|c|c|c|c|}
\hline & $\begin{array}{c}\mathrm{H} 8 \\
\text { (Gua) }\end{array}$ & $\begin{array}{c}\mathrm{H} 1^{\prime} \\
\text { (Rib) }\end{array}$ & $\begin{array}{c}\mathrm{H} 1 \\
\text { (Man) }\end{array}$ & $\begin{array}{l}\text { H2' } \\
\text { (Rib) }\end{array}$ & $\begin{array}{l}\mathrm{H}^{\prime} \\
\text { (Rib) }\end{array}$ & $\begin{array}{l}\mathrm{H}^{4} \\
\text { (Rib) }\end{array}$ & $\begin{array}{l}\mathrm{H} 5 / 5^{\prime} \\
\text { (Rib) } \\
\end{array}$ & $\begin{array}{c}\mathrm{H} 2 \\
\text { (Man) }\end{array}$ & $\begin{array}{l}\mathrm{H} 3, \mathrm{H} 5, \\
\mathrm{H6} 6^{\prime} / \mathrm{H} 6^{\prime \prime} \\
\text { (Man) } \\
\end{array}$ & $\begin{array}{c}\mathrm{H} 4 \\
\text { (Man) }\end{array}$ \\
\hline Asp-GEF (STD) & 92 & 100 & 23 & n.d. & 31 & 39 & 39 & 18 & 15 & 24 \\
\hline $\begin{array}{l}\text { (Asp-GeF)-(Asp } \\
- \text { GeF-DN) } \\
\text { (STDD) }\end{array}$ & 61 & 100 & 37 & n.d. & 40 & 40 & 22 & 22 & 15 & 21 \\
\hline $\begin{array}{l}\text { (Asp-GeF)- } \\
\text { (human-GeF) } \\
\text { (STDD) }\end{array}$ & 95 & 100 & 37 & n.d. & 31 & 39 & 24 & 18 & 19 & n.d. \\
\hline
\end{tabular}

